\title{
Identity, Structure and Compositional Analysis of Adjuvanted Vaccines
}

\author{
Kristen Kalbfleisch ${ }^{1 \dagger}$, Moriam Ore 1,2, Sasmit Deshmukh 1,3, Wayne Williams ${ }^{1}$, Ibrahim \\ Durowoju ', Jessica Duprez ', Carmen Mei 1, Sylvie Morin 2, Joan Bevilacqua ', Bruce Carpick 1, \\ and Marina Kirkitadze 1,* \\ 1 Analytical Research and Development, Sanofi Pasteur Ltd., 1755 Steeles Avenue West, Toronto, Ontario, \\ Canada; Moriam.Ore@sanofi.com, Sasmit.Deshmukh@sanofi.com, Wayne.Williams@sanofi.com, \\ Ibrahim.Durowoju@sanofi.com, Jessica.Duprez@sanofi.com, Carmen.Mei@sanofi.com, \\ Bruce.Carpick@sanofi.com, Marina.Kirkitadze@sanofi.com \\ 2 Department of Chemistry, York University, 4700 Keele Street, Toronto, Ontario, Canada; \\ seunore@yorku.ca, smorin@yorku.ca \\ 3 SGS Canada, Biopharmaceutical Services, 6490 Vipond Drive, Mississauga, Ontario, Canada \\ 4 tFlair Flexible Packaging Corporation, 410072 Ave SE, Calgary, AB T2C 2C1, Canada \\ kristen_kalbfleisch@hotmail.com \\ * Correspondence: marina.kirkitadze@sanofi.com; Tel.: +1 416-667-2095
}

\begin{abstract}
Background: Traditionally, complex biological products such as vaccines presented unique challenges to implementation of even rudimentary characterization packages; thus, the product was defined almost exclusively by its manufacturing process. The advances in technology and analytical tools allowed the application of more comprehensive characterization packages for products such as adsorbed combination vaccines, which contain several antigens in a single formulation to protect against more than one disease, and may contain adjuvants and excipients. Aluminum phosphate $\left(\mathrm{AlPO}_{4}\right)$ is a well-established adjuvant for enhancing the uptake of vaccines and to induce robust immunity against pathogens. During manufacturing, adjuvant is mixed with protein antigens which may in turn impact their higher order structure and stability. 2) Methods: To study the structural changes of protein antigens after adsorption several analytical tools including DLS, FTIR, Fluorescence, LD, and SEM were used. 3) Results: the $\mathrm{AlPO}_{4}$ adjuvant suspension consists of small submicron particles that form a continuous porous surface. Secondary structure alpha-helix and beta-sheet content of DT and TT increased after adsorption to AlPO adjuvant, whereas no significant changes were noted for other protein antigens. Interactions were noted between $\mathrm{AlPO}_{4}$ adjuvant and DT, TT, and FHA. 4) Conclusions: here we report for the first time the use of SEM for the visualization of adsorbed multivalent vaccine components. A unique signature profile detected for each multivalent vaccine by FTIR can be used as a lean in-process test to verify vaccine product composition and identity prior to filling.
\end{abstract}

Keywords: adsorbed vaccines, identity, protein conformation, particle sizing, FTIR, SEM, Fluorescence

\section{Introduction}

Advances in analytical tools now permit significant characterization of complex biological products such as protein vaccine antigens, which traditionally have been defined primarily by their manufacturing processes. The application of extensive characterization packages can now extend beyond simply characterizing the purified proteins, to include product intermediates [1,2], and adsorbed protein drug substances and adjuvanted vaccine formulations [3,4,5,6]. As discussed previously [7], characterization of vaccine attributes at both the drug substance and drug product stages have progressively higher criticality with respect to product supply, safety and immunogenicity. For vaccines, this encompasses not only protein antigens, but also adjuvants, and adjuvanted and multivalent product formulations. Factors that can affect safety and efficacy critical 
quality attributes and critical material attributes may include, but are not limited to, protein adsorption and conformation, size distribution and morphology of adsorbed drug substances (DS). Presented here, to assess these attributes, are several of analytical tools with the capability of characterizing multivalent vaccines and their components, as well as lot-to-lot consistency. The principle applied here is that the quality of subsequent batches is the consequence of the strict application of a quality system and of a consistent production of batches which can be demonstrated using state-of-the-art and non-animal methods [8].

Many reports in the literature demonstrate that protein adsorption to an adjuvant can alter its conformation $[4,6,9,10]$, and either stabilize [11], destabilize $[3,9,12]$, or show no effect [5] on conformation. This highlights the importance of analytical tools capable of monitoring these possible changes in protein antigens throughout the manufacturing process.

Multivalent vaccines offer better protection against certain diseases such as pertussis [13], and manufacturing of combination products with the same immunogenicity and safety profile as each of its individual component vaccines is a considerable challenge [14]. Development of combination vaccines requires a careful assessment and selection of adjuvant(s) and process steps including formulation of the intermediates and final product. Furthermore, there may be physicochemical or immunological interference between any or all of the components [15].

To provide a more comprehensive analysis of current manufacturing processes, samples of intermediate pre-adsorbed protein antigens, adsorbed drug substances, and drug products were examined using a panel of methods. These included dynamic light scattering (DLS), laser diffraction (LD), scanning electron microscopy (SEM), Fourier transform infrared (FTIR) spectroscopy, and intrinsic fluorescence (IF) spectroscopy. These non-routine characterization tests were applied for the purpose of product knowledge.

Particle size can be an indication of both process consistency and product stability, and can be a quality attribute used in the characterization of vaccine and vaccine components [16]. DLS was utilized to characterize the size of pre-adsorbed protein antigens, while LD was applied to particle sizing of adjuvant and adjuvanted dug substances.

As antigen protein conformation may affect the presentation of epitopes, the effect of adjuvantation on protein higher order structure was analyzed. FTIR was utilized to measure secondary structure content, and IF to examine tertiary structure conformation.

With the objective of a comprehensive characterization of multivalent vaccines and their components, a novel SEM approach to the visualization of adjuvant size and morphology was developed. Use of low vacuum SEM imaging mode allows characterization of non-conductive biomaterials [15]. This allowed an investigation of the effect of adsorbed proteins on adjuvant morphology and packing density of the suspension that in turn could be used to gain product knowledge and characterize adjuvantation step of the manufacturing process.

Finally, although various multivalent vaccines may contain similar antigen profiles, minor variations in their composition or formulation may be detected by a sufficiently sensitive and selective method. FTIR was found to be capable of detecting a signature spectrum, not only for individual adsorbed monovalent drug substances, but also for several multivalent vaccine drug products. Thus, FTIR can be used to identify very similar drug products.

\section{Results}

\subsection{Particle size, morphology, and composition}

Size distribution profiles and particle size of vaccine intermediates were measured using DLS and LD. DLS was used for pre-adsorbed samples while LD was used for adjuvants and adsorbed vaccines (Figure 1 and A1). The size distribution profiles as determined by DLS for each of the pre-adsorbed antigens are shown in Figure 1. These antigens will ultimately be formulated into a multivalent vaccine with protection against Pertussis, Diphtheria and Tetanus. On an average, particle sizes ranged from 10 to $200 \mathrm{~nm}$, and show the diversity of size from antigen to antigen. Each showed a unique distribution profile (see Table A1 for particle size values). 

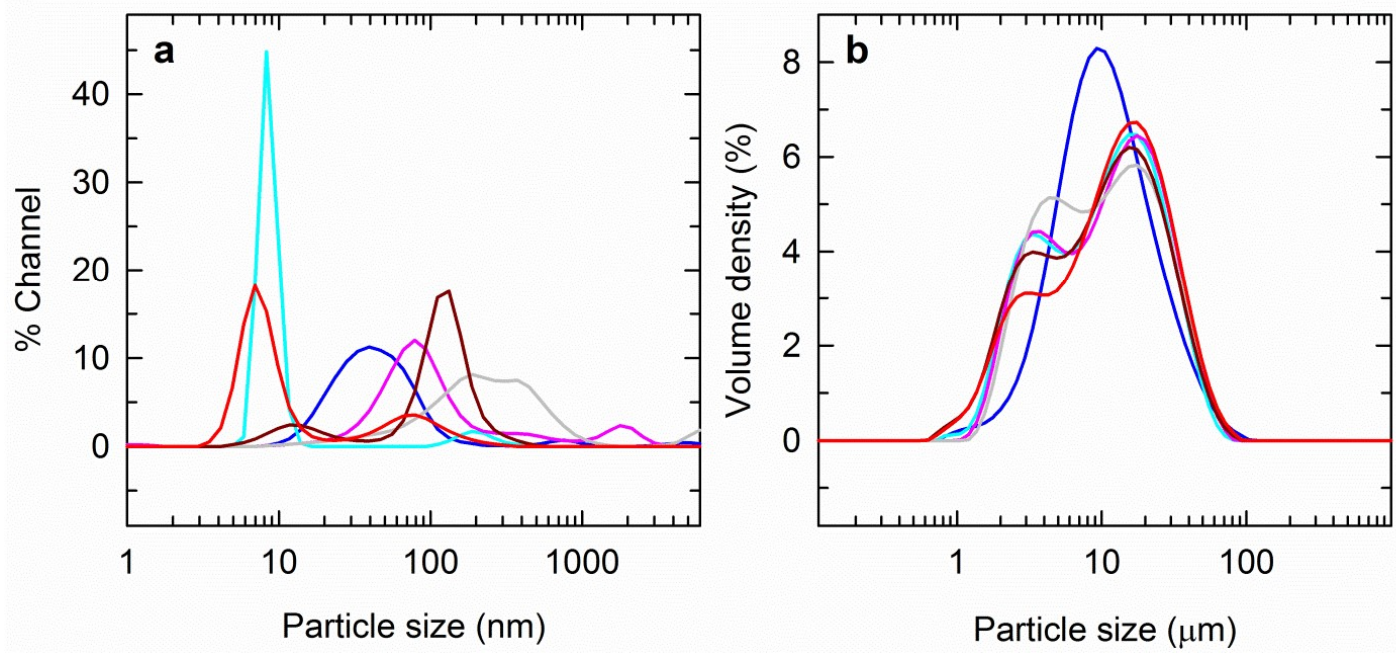

Figure 1: Particle size distribution of (a) pre-adsorbed and (b) adsorbed protein antigens by DLS and LD respectively. Representative traces include PRN (cyan), DT (red), FHA (blue), FIM (pink), TT (brown), and PT (grey) antigens.

$\mathrm{AlPO}_{4}$ was used as an adjuvant in the adsorbed form of the antigen proteins, and its particle size (Dv50 value) was around $13 \mu \mathrm{m}$ (Figure A1a).

The size distribution profiles depicted in Figure $1 \mathrm{~b}$ were representative of the three lots of each product analyzed. With the exception of adsorbed FHA, each monovalent adsorbed antigen profile showed two major peaks and a broad size distribution ranging from approximately $1-100 \mu \mathrm{m}$. By contrast, adsorbed FHA showed one major peak and a narrower size distribution. Final drug product (data not shown) that includes all six adsorbed protein antigens was similar in size distribution to most of the monovalent adsorbed antigens.

In addition to particle size, the previously unexplored characteristic of particle morphology was visualized for the first time for an $\mathrm{AlPO}_{4}$ adjuvant suspension, as well as for each of the adsorbed monovalent antigens. Panel b in Figure A1 depicts a low vacuum SEM image of AlPO adjuvant, most prominently highlighting the formation of irregularly shaped agglomerates comprised of smaller particles. In Figure 2, six $\mathrm{AlPO}_{4}$ adsorbed monovalent antigens are compared using low vacuum SEM, in which finer features of the surface can be visualized, compared with conventional SEM [17]. 

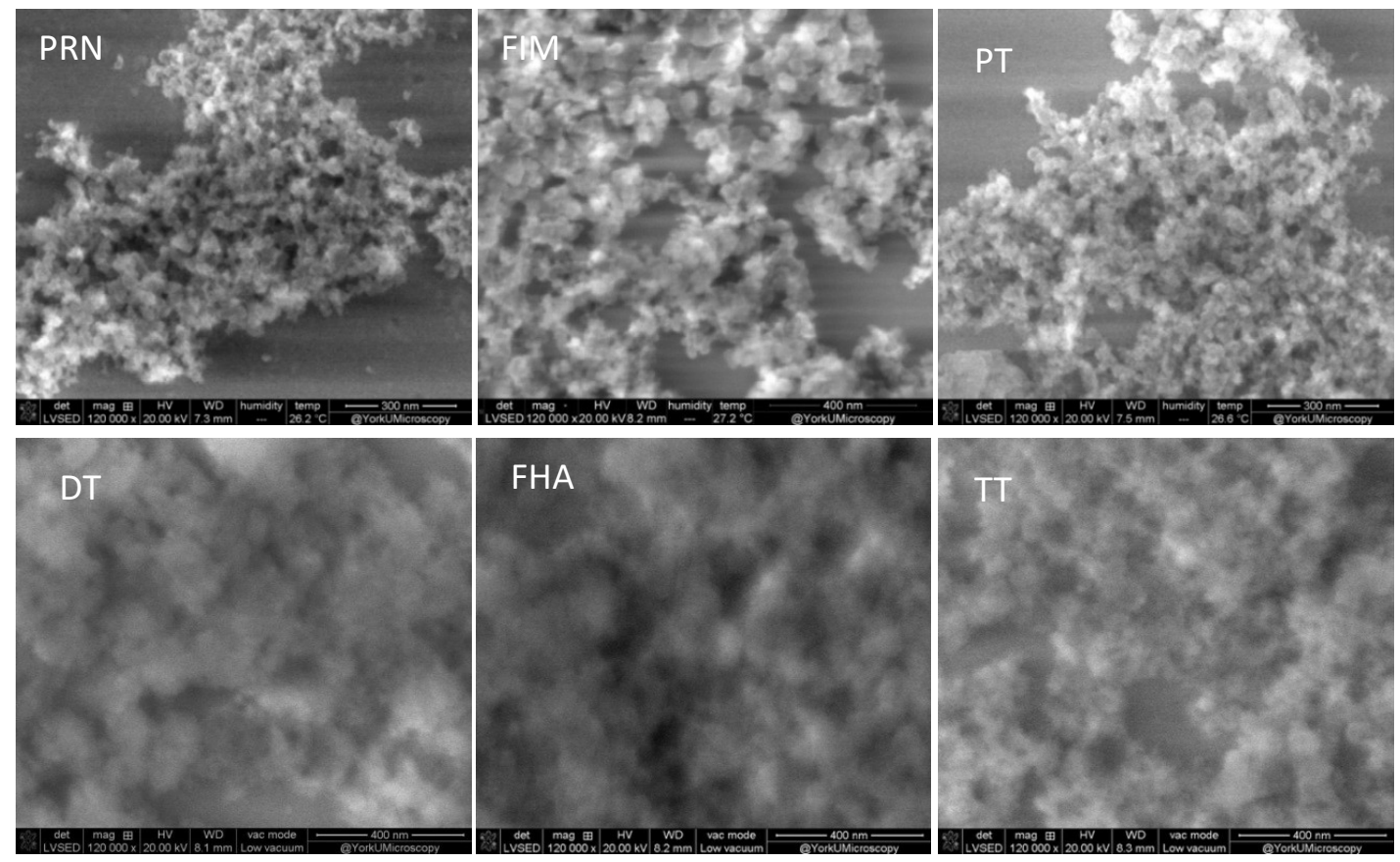

Figure 2: Low vacuum SEM images of $\mathrm{AlPO}_{4}$ adsorbed antigens.

As shown in Figure 2, adsorbed protein samples and $\mathrm{AlPO}_{4}$ adjuvant were similar in morphology when imaged in low vacuum mode, and no changes to adjuvant were observed after antigen adsorption.

\subsection{Secondary Structure}

As previously discussed, proteins adsorbed on the surface of adjuvant particles may undergo conformational changes. Higher order structural changes following $\mathrm{AlPO}_{4}$ adsorption were characterized by different spectroscopic methods: FTIR (secondary structure), and IF (tertiary structure). FTIR spectroscopy was used to probe the conformational changes associated with adsorption by monitoring shifts in secondary structure from pre-adsorbed to adsorbed in purified monovalent antigens (Figure 3). Table A2 indicates the changes in alpha helix and beta sheet content upon adsorption.

An increase in both alpha-helix and beta-sheet content were observed for DT and TT upon adsorption to $\mathrm{AlPO}_{4}$ (Table A2). However, for PRN, FIM and FHA the changes detected were within the experimental error and hence deemed insignificant. While pre-adsorbed and adsorbed PT antigen did not show any spectral change (Figure 3). FTIR spectra for all six adsorbed monovalent antigens are presented, and supplemented with the second derivative spectra to highlight regions of variability. All protein antigens characterized, except PT, also showed a broad peak around 1078 $\mathrm{cm}^{-1}$ for $\mathrm{AlPO}_{4}$ adjuvant in the adsorbed form. Some small changes were also observed in protein backbone and sidechain around 1400 and $1453 \mathrm{~cm}^{-1}$ as a result of adjuvantation. 


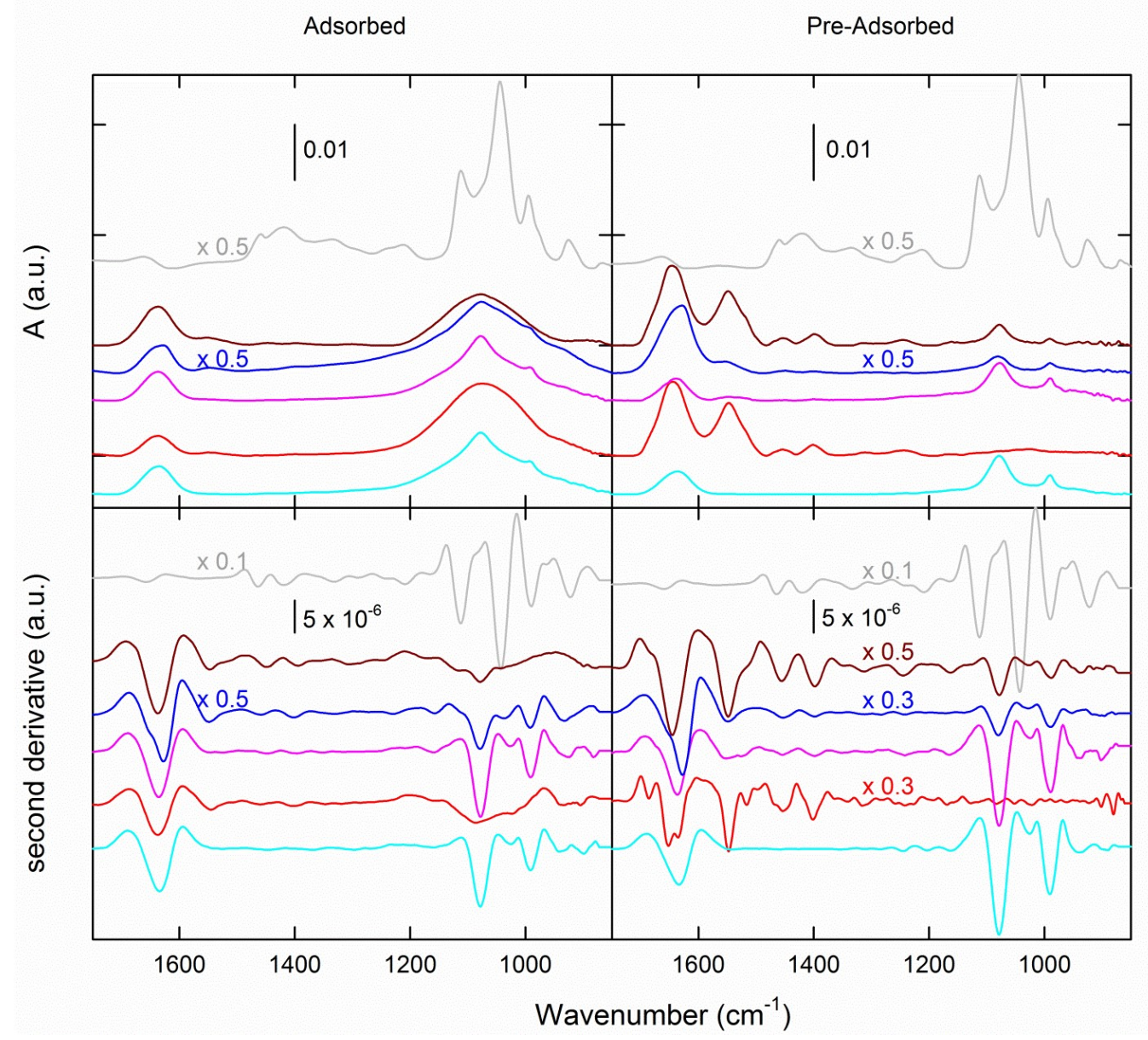

Figure 3: Representative FTIR spectra (top panels) and their calculated second derivatives (bottom panels) for all adsorbed (left panels) and pre-adsorbed (right panels) antigens. Some of measured spectra and calculated first derivative spectra are rescaled due to high signal intensity. Representative traces include PRN (cyan), DT (red), FHA (blue), FIM (pink), TT (brown), and PT (grey) antigens.

All drug substances, as well as the final multivalent product samples (Figure 3 and 4) showed unique spectral features. As shown in the upper left panel in Figure 3, similar spectral features are observed in FHA, FIM and PRN, whereas by contrast, DT and TT were similar to some extent. In cases where unambiguous distinction is difficult by comparing spectra, calculated 2nd derivative spectra can elaborate additional spectral information, as shown in the lower panel of Figure 3 . In this analysis, the differences emerge within the amide I and II regions for each of the tested drug substances in the pre-adsorbed versus adsorbed forms. This region highlights the changes in $\beta$-sheet, turns and $\alpha$-helices at approximately 1624, 1676 and $1654 \mathrm{~cm}-1$, respectively. Secondary structural content may be influenced by adsorption to $\mathrm{AlPO}_{4}$ as a result of changes to the local environment of the antigens which can also be detected by the shifts in the FTIR peak positions. The low frequency region (between approximately 1076 and $990 \mathrm{~cm}-1$ ) consists mainly of contributions from adjuvant and phosphate buffer.

\subsection{Identity}

The combination or multivalent vaccine products Quadracel ${ }^{\mathrm{TM}}$, Pentacel ${ }^{\circledR}$, and Pediacel ${ }^{\circledR}$ [18-20] all contain $\mathrm{AlPO}_{4}$ as an adjuvant and have many antigens in common. As a result, the spectral features of these combination products are quite similar (Figure 4), yet small but detectable differences were observed. For instance, the peak representative of the P-O stretch (around 1079 $\mathrm{cm}^{-1}$ ) had higher absorbance in Quadracel ${ }^{\mathrm{TM}}$ when compared to Pentacel ${ }^{\circledR}$ or Pediacel ${ }^{\circledR}$, the latter 
showing a shift in this peak (to $1083 \mathrm{~cm}^{-1}$ ). Another spectral difference was observed at $1420 \mathrm{~cm}^{-1}$ in Pentacel ${ }^{\circledR}$ and Quadracel ${ }^{\mathrm{TM}}$, where both showed a broad shallow peak in contrast to the sharper peak detected at $1414 \mathrm{~cm}^{-1}$ in Pediacel ${ }$.

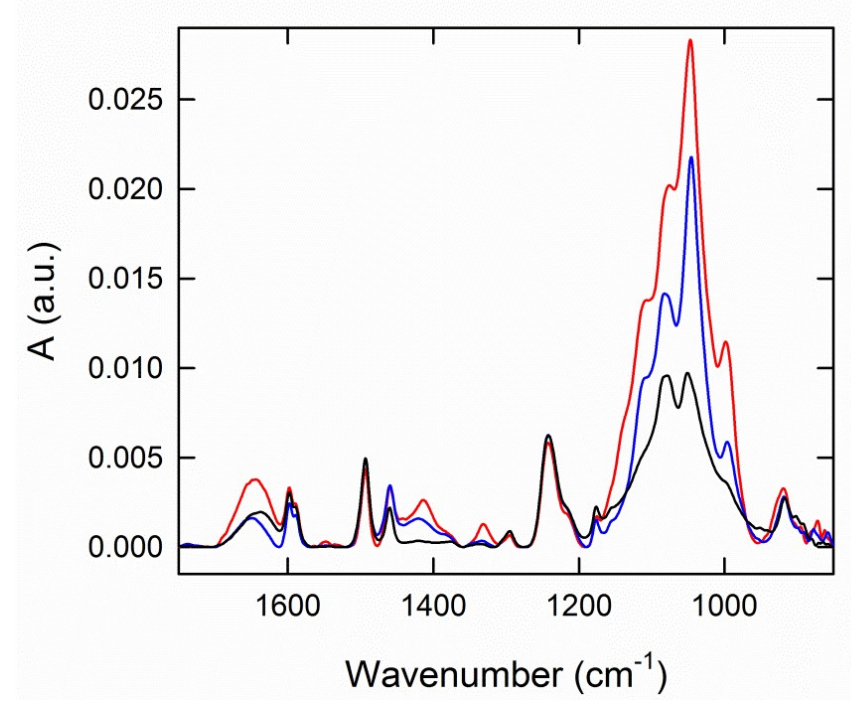

Figure 4. Overlay of FTIR spectra for Pediacel ${ }^{\circledR}$ (red trace), Pentacel ${ }^{\circledR}$ (blue trace) and Quadracel ${ }^{\mathrm{TM}}$ (black trace).

\subsection{Tertiary Structure}

Intrinsic fluorescence spectroscopy (IF) was used to probe effect of adsorption on tertiary structure of the proteins. IF emission spectra of DT and TT revealed that adsorbed form of the protein has hypsochromic shift in tryptophan fluorescence emission as compared to pre-adsorbed antigens (Figure 5). 


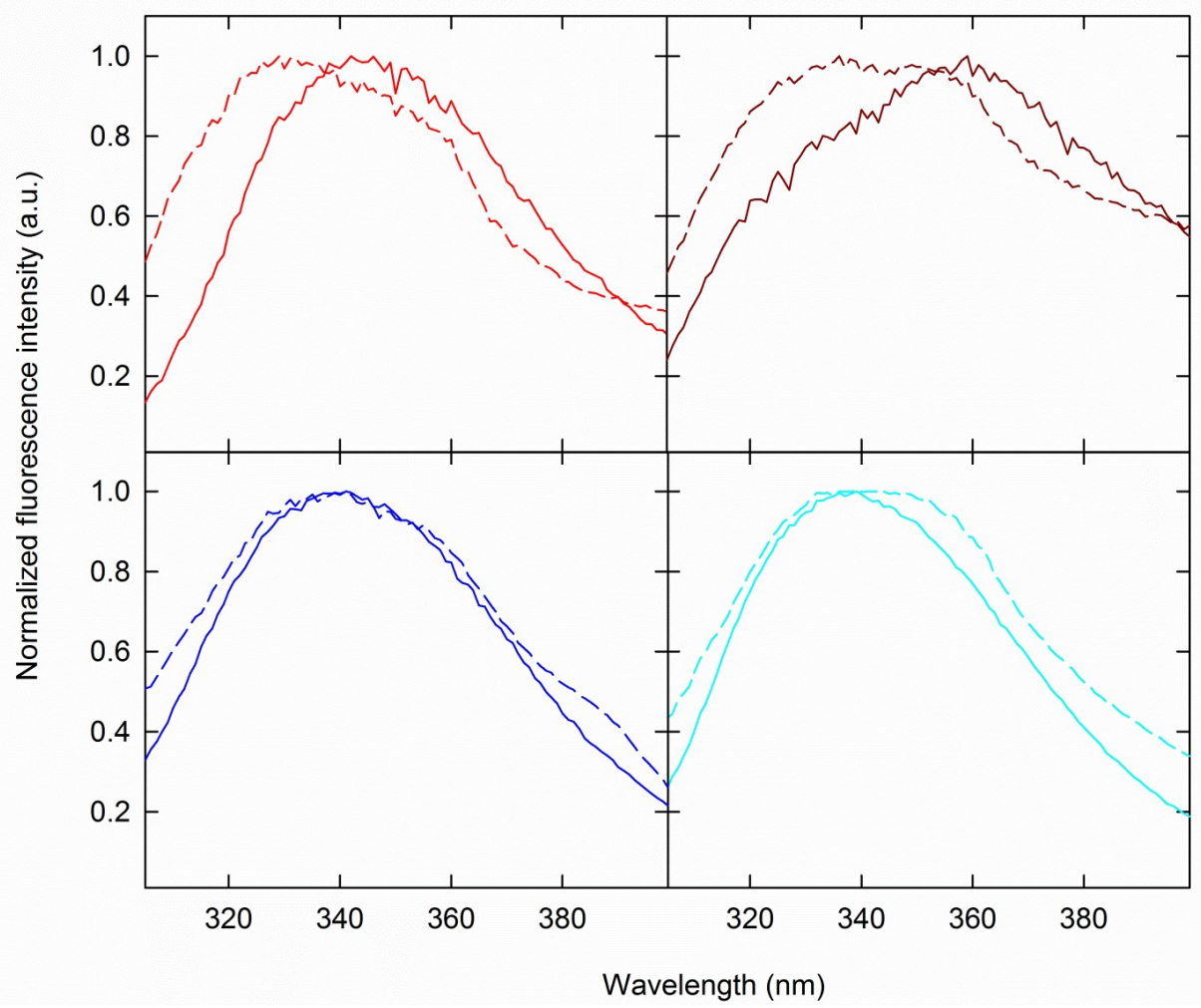

Figure 5. Intrinsic fluorescence emission spectra of DT (red), TT (brown), FHA (blue), and PRN (cyan) antigens in pre-adsorbed (solid traces) and adsorbed (short dashed traces) forms.

FHA and PRN showed no significant shift whereas FIM and PT did not show fluorescence emission signal in either form.

\section{Discussion}

Vaccines are complex formulations containing multiple components such as protein antigens, adjuvants, excipients, stabilizers etc., and hence they form complex interations within the matrix. Therefore it is imperative to perform identity, compositional and structural analysis as a mean of quality control as well as to gain product knowledge. This study focuses on these aspects of the vaccine components and products through set of biophysical methods.

The results from particle size distribution suggest that $\mathrm{AlPO}_{4}$ adjuvant primarily affects the overall size of the adsorbed protein antigens (Figure 1 and A1a). It also appears that the majority of particle size distribution profiles of the adsorbed protein antigens have some variability from particle size of $\mathrm{AlPO}_{4}$ as a result of adjuvantation (Figure $1 \mathrm{~b}$ and A1, and Table A1). Apparently, particle size is important in the uptake of particles by antigen presenting cells [16], and the size of $10 \mu \mathrm{m}$ is optimal [16]. This is in agreement with the particle sizes (Dv50 values) of adsorbed protein antigens found in this study (Table A1). The adjuvant suspension consists of small submicron particles that form continuous porous surfaces, and dense surface texture, which may impact antigen adsorption (Figure A1b) and therefore there is some variability for particle size distribution of adsorbed protein antigens.

SEM images demonstrate that $\mathrm{AlPO}_{4}$ suspension and adsorbed proteins consist of small submicron particles that form a continuous porous surface (Figure 3 and A1b). The approximate overall size of these particles is of $\sim 4-5 \mu \mathrm{m}$ as measured by SEM and $\sim 8-14 \mu \mathrm{m}$ as shown by LD. These differences are due to experimental conditions, such as hydration level of the adjuvant 
suspension, presence of vacuum for SEM measurements. As shown internally by Electrochemiluminescence and ELISA assays (data not shown), DT, TT, and FHA show high \% adsorption (about 90\%) to $\mathrm{AlPO}_{4}$, whereas \% adsorption of PRN, PT, and FIM is low (below 30\%). Adjuvant particles with DT, TT, and FHA appear larger in size possibly denser due to interaction between proteins and adjuvant surface. The adjuvant appears to be coated with the protein (DT, TT, or FHA) making the surface less conductive, and resulting in less sharp images.

FTIR spectroscopy was used to probe secondary structural changes as a result of adjuvantation due to its ability to measure adjuvanted samples using ATR crystal. In FTIR spectra, individual peaks represent vibrational modes of the molecules under study and the alteration in the local environment of these molecules is detected by shifts in the peaks or the appearance or disappearance of certain peaks. This information was used while acquiring and analyzing spectra of these vaccine components. Drug substances which primarily consist of single antigens can be characterized using FTIR before and after adjuvant formulation. All of the vaccine antigens tested are purified proteins and thus share some fundamental FTIR spectral features. Moreover, the degree of adsorption to $\mathrm{AlPO}_{4}$ adjuvant may differ among antigens due to concentration, pI, or other factors and this may complicate the analysis of spectral features in adsorbed samples. Besides PT all other protein antigens showed spectral changes from pre-adsorbed to adsorbed formulations (Figure 3). This suggests that PT does not have surface adsorption to $\mathrm{AlPO}_{4}$ which is also in agreement with SEM data. Besides PT and PRN all other protein antigens in the pre-adsorbed form have amide II signal which disappears due to adjuvantation, this indicates structural changes involving amide II region. To summarize, FTIR can be used as lean technique to verify identity of the bulk drug substance prior to formulation and also to gain knowledge about changes to protein antigens as a result of adsorption.

As demonstrated in a recent study [21], the FTIR spectra of both adsorbed monovalent antigens and multivalent vaccine products (Figure 3 and Figure 4) showed rich information that can be recorded as a spectral fingerprint for each tested sample allowing FTIR spectroscopy to be used as a lean technique to verify the bulk drug substance identity prior to formulation, and in-process test to verify vaccine product identity prior to filling.

Besides changes in the secondary structure of the protein antigen, tertiary structure also showed some changes as result of adjuvantation. Intrinsic fluorescence emission spectra showed band broadening in the adsorbed antigens likely due to altered solvent interactions with each fluorophore (Figure 5). The overall hypsochromic shift in adsorbed DT and TT indicate that tryptophan residues are more buried and have less solvent access, which could indicate these proteins are more folded than their pre-adsorbed forms or these residues are shielded by the adjuvant surface. 
9 of 13

\title{
4. Materials and Methods
}

Reagents and Materials

All samples examined in this study were manufactured in-house, including adjuvant, pre-adsorbed and adsorbed protein samples (i.e. drug substances), and final vaccine products.

\section{Dynamic Light Scattering (DLS)}

All measurements of particle size distribution of pre-adsorbed antigens were performed using a Nanotrac 150 instrument (Betatek, PA, USA). Dynamic light scattering (DLS) measures the diameter of particles based on the principle of diffusion through the solvent due to Brownian motion, by recording the fluctuations in the intensity of the scattered light. The particle size measurement is an intensity averaged distribution using the Stokes-Einstein equation:

\author{
$\mathrm{d}(\mathrm{H})=\mathrm{kT} / 3 \pi \eta \mathrm{D}$ \\ where, \\ $\mathrm{d}(\mathrm{H})$ : hydrodynamic diameter of the particle \\ k: Boltzmann's constant \\ T: absolute temperature \\ $\eta$ : solvent viscosity \\ D: diffusion coefficient (determined from Brownian motion of the particle).
}

\section{Laser Diffraction (LD)}

All measurements of particle size distribution of adjuvant, adsorbed antigens and multivalent vaccine products were performed using a Mastersizer 3000 instrument (Malvern Instruments Ltd., UK), operating in a dynamic range of 0.01 to $3500.00 \mu \mathrm{m}$. Particle size distributions in solutions and suspensions were quantitatively determined by measuring the angular variation in intensity of light scattered from a laser beam passing through a dispersed particulate sample. Large particles scatter light at smaller angles than smaller particles, relative to the beam. The angular scattering intensity data is then analyzed to calculate the size of the particles responsible for creating the scattering pattern, using Mie theory of light scattering. The reportable value is Derived Diameter (Dv), which is the particle size (in $\mu \mathrm{m}$ ) for a specific percentile of the cumulative size distribution. Particles were measured using the built-in "non-spherical" option within the software, and the average Dv10, Dv50 and Dv90 values of 5 measurements are reported. These are the mass median diameters at which the given percent $(10,50$ or 90$)$ of the sample is smaller than the reported value. Re-plotting was performed using SigmaPlot.

\section{Fourier Transform Infrared (FTIR) Spectroscopy}

Fourier transformed infrared spectroscopy (FTIR) is a technique based on absorption of infrared photons that have energies similar to the vibrational energies of molecular bonds. Since vibrational energy transitions are quantized, a bond can be caused to vibrate if it absorbs a photon with a frequency equal to its natural vibrational frequency. This absorption of IR photons forms the basis of IR spectroscopy. The IR spectrum as a function of wavenumber is calculated with the use of Fourier Transformation. FTIR spectroscopy was performed using a Vertex 70 FTIR Spectrometer (Bruker Optics, Bremen, Germany), equipped with a cryogenically-cooled MCT (mercury-cadmium-telluride) detector and a BioATRII sampling accessory. A sample volume of 20 $\mu \mathrm{L}$ was loaded onto the sample cell and the spectra were collected at a resolution of $0.4 \mathrm{~cm}^{-1}$ at $25^{\circ} \mathrm{C}$ with a wavenumber accuracy of $0.01 \mathrm{~cm}-1$ at $2000 \mathrm{~cm}^{-1}$. The samples were allowed to stabilize for 1 minute on the ATR crystal. Background (Milli-Q water) and sample measurements were conducted with each reported measurement representing an average of 200 scans. Data acquisition and analysis were performed using the OPUS 6.5 software (Bruker Optics, Bremen, Germany). OPUS automatically subtracts the background signal from the sample to produce the spectrum for the analyte. All measurements were carried out at $25^{\circ} \mathrm{C}$ using a Haake DC30/K20 temperature controller (Karlsruhe, Germany). After acquiring the FTIR spectra, the baseline was corrected by removing the 
scattering signal using the OPUS software. Quant2 software (Bruker Optics) was used to estimate secondary structure with an error of $5.5 \%$ for alpha-helix content and $4.4 \%$ for beta-sheet content. The second derivative spectrum was generated using the Savitzky-Golay algorithm which allowed simultaneous smoothing of the spectrum. Arithmetic manipulations and re-plotting were performed using SigmaPlot.

Scanning Electron Microscopy (SEM)

SEM is an analytical tool for elucidating the morphology and size of various materials. It provides high-resolution images by irradiation with a high energy electron beam across a sample surface and detecting primary and secondary electron signals. The most common detectable signal for topographical image is the secondary electron, which is produced by the ejection of core electrons from atoms at the sample surface. All measurements were performed using FEI Quanta 3D SEM (ThermoFisher Scientific, United States) in the Imaging Facility at York University. Low Vacuum SEM mode was used to image the dried adjuvanted samples and was accomplished by centrifugation of the sample at $6000 \mathrm{rpm}$, followed by removal of the supernatant. It should be noted that the sample preparation is essential for the resolution of adjuvant morphology, and this SEM method was optimized accordingly. $\mathrm{NaCl}$, a residual of adjuvant production process, may interfere with SEM characterization of the microstructure, and therefore, samples were rinsed prior to analysis. Pellets were then rinsed 3 times with LC-grade water. The rinsed pellets were then immobilized by smearing a small amount of the adjuvant suspension on a glass microscope slide. The samples were imaged using a low vacuum secondary electron detector.

\section{Intrinsic Fluorescence spectroscopy (IF)}

IF spectroscopy was performed using Varian Cary Eclipse spectrophotometer (Agilent Technologies, Santa Clara, CA, USA). Intrinsic fluorescence, a dye-free method to evaluate changes in aromatic amino acid residues (fluorophores) within proteins, was used to probe changes in the local environment as a result of adsorption onto the surface of $\mathrm{AlPO}_{4}$ adjuvant. All protein samples were excited at $285 \mathrm{~nm}$ and emission spectra were recorded in 300 to $400 \mathrm{~nm}$ region using multi-cell holder accessory of Cary eclipse. Measuring parameters such as slit width were optimized for each sample to obtain maximum fluorescence intensity.

\section{Conclusions}

To summarize, we applied an ensemble of methods to the analysis of pre-adsorbed and adsorbed vaccine antigens, drug substances, and drug products. The product attributes analyzed included particle size, particle morphology, protein higher order structure, and vaccine composition. As shown by SEM, the $\mathrm{AlPO}_{4}$ adjuvant suspension consists of small submicron particles that form a continuous porous surface. As shown by FTIR, secondary structure alpha-helix and beta-sheet content of DT and TT increased after adsorption to $\mathrm{AlPO}_{4}$ adjuvant, whereas no significant changes were noted for other protein antigens besides structural changes within the amide region. Similarly, SEM showed strong interactions between $\mathrm{AlPO}_{4}$ adjuvant and DT, TT, and FHA. Finally, FTIR spectroscopy demonstrated applicability for a direct method capable of identifying final drug product without desorption using a unique spectrum (fingerprint) generated by a combination of protein antigens and excipients. Although multivalent vaccines can appear to be very similar in formulation, the addition of Haemophilus influenzae conjugate component and excipients in the formulation results in a unique signature profile for each product tested thus far.

In summary, the ensemble of methods employed in this study represents a non-traditional approach for structural and compositional analysis of complex biological products, namely multivalent adjuvanted vaccines. In the case of LD and FTIR, information about particle size, protein structure, and product composition can be obtained on the entire product without complicated or destructive sample manipulation steps such as antigen desorption. Low-vacuum SEM represents a unique approach for direct visualization of the morphology of the adjuvant surface in the presence of adsorbed vaccine antigens. 
Author Contributions: Conceptualization, M.K., B.C., J.B.; Validation, M.O., C.M., S.M., and J.D.; Investigation, S.D., K.K., I.D., W.W., J.D., C.M.; Resources, M.K.; Writing-Original Draft Preparation, K.K., I.D., M.K., W.W., S.D.; Writing-Review \& Editing, B.C., K.K., M.K., I.D., J.B., J.D., S.D., C.M., S.M., M.O.; Supervision, M.K.; Project Administration, M.K., B.C., J.B.; Funding Acquisition, S.M, M.O., M.K.

Funding: This research was funded by NSERC grant number [EGP 507493-2017].

Acknowledgments: Sylvie Morin, Moriam Ore, and Marina Kirkitadze would like to thank the Government of Canada for providing assisting funds through an NSERC engage grant [EGP 507493-2017]. Sylvie Morin and Moriam Ore acknowledge the support of York University for this research.

Conflicts of Interest: he authors declare no conflict of interest. Kristen Kalbfleisch, Sasmit Deshmukh, Wayne Williams, Ibrahim Durowoju, Jessica Duprez, Carmen Mei, Joan Bevilacqua, Bruce Carpick, and Marina Kirkitadze are employees of Sanofi Pasteur, and Sylvie Morin and Moriam Ore are the employees of York University and have no other relevant affiliations or financial involvement with any organization or entity with a financial interest in or financial conflict with the subject matter or materials discussed in the manuscript. Thus includes employment, consultancies, stock ownership or options, or royalties.

\section{Abbreviations}

$\begin{array}{ll}\text { SEM } & \text { Scanning Electron Microscopy } \\ \text { DLS } & \text { Dynamic Light Scattering } \\ \text { FTIR } & \text { Fourier Transform Infrared Spectroscopy } \\ \text { LD } & \text { Laser Diffraction } \\ \text { IF } & \text { Intrinsic Fluorescence }\end{array}$

\section{Appendix A}

Supplementary materials
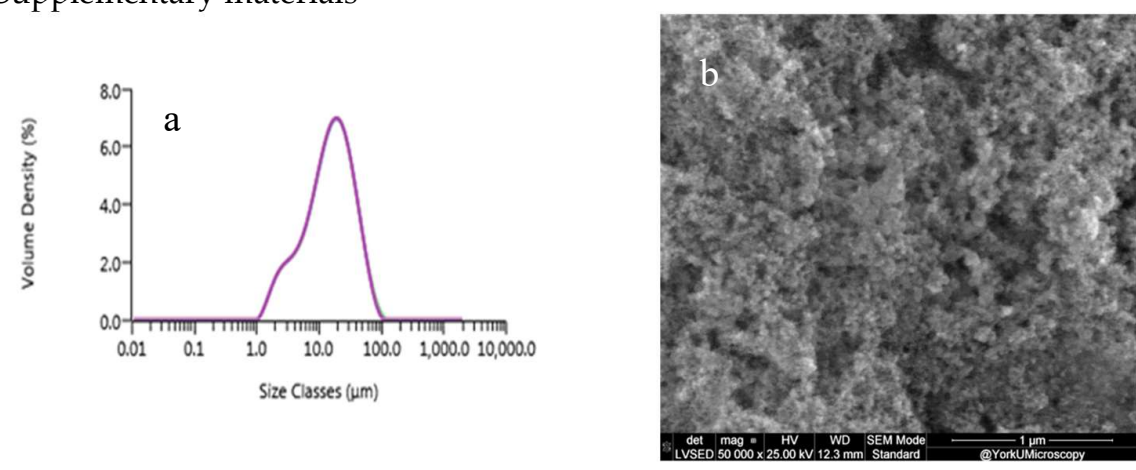

Figure A1: LD size distribution profile (a) and SEM morphology (b) for $\mathrm{AlPO}_{4}$ adjuvant

Table A1: Particle size distribution of pre-adsorbed (DLS) and adsorbed (LD) protein antigens

\begin{tabular}{|c|c|c|c|c|}
\hline \multirow[t]{2}{*}{ Antigen name } & \multirow{2}{*}{$\begin{array}{l}\text { Hydrodynamic diameter by DLS, } \\
\mu \mathrm{m}\end{array}$} & \multicolumn{3}{|c|}{ Size distribution by $L D, \mu \mathrm{m}$} \\
\hline & & Dv10 & Dv50 & Dv90 \\
\hline PRN & 0.008 & 2.6 & 11 & 30 \\
\hline DT & 0.07 & 2.5 & 12 & 33 \\
\hline TT & 0.12 & 2.4 & 10 & 31 \\
\hline PT & 0.18 & 2.9 & 10 & 32 \\
\hline FIM & 0.078 & 2.7 & 11 & 32 \\
\hline FHA & 0.04 & 4 & 10 & 28 \\
\hline
\end{tabular}


Table A2: Alpha-helix and Beta-sheet content of protein antigens.

\begin{tabular}{|c|c|c|}
\hline Sample & Alpha-helix, $\%$ & Beta-sheet, $\%$ \\
\hline Pre-adsorbed PRN & & 47 \\
AlPO4 Adsorbed PRN & & 49 \\
Pre-Adsorbed DT & 47 & 37 \\
AlPO4 Adsorbed DT & 53 & 48 \\
Pre-Adsorbed TT & 28 & 36 \\
\hline AlPO4 Adsorbed TT & 47 & 46 \\
Pre-Adsorbed FIM & & 46 \\
AlPO 4 Adsorbed FIM & 48 \\
Pre-Adsorbed FHA & 50 \\
AlPO4 Adsorbed FHA & & 52 \\
\hline
\end{tabular}

*Note: PRN, FHA, and FIM are predominantly beta-sheet proteins that also contain loops and unfolded regions.

\section{References}

1. Tung, J.-S.; Gimenez, J.; Przysiecki, C.T.; Mark, G. Characterization of Recombinant Hepatitis B Surface Antigen Using Surface Plasmon Resonance. Journal of Pharmaceutical Sciences 1998, 87(1), 76-80.

2. Durowoju, I.; Bhandal, K.; Hu, J.; Carpick, B.; Kirkitadze, M. Assessment of Tertiary Structure of Protein Vaccine Antigens by Differential Scanning Calorimetry. Journal of Visualized Experiments 2017, 121, e55262. Video: http://www.jove.com/video/55262; doi:10.3791/55262.

3. Vessely, C.; Estey, T.; Randolph, T.W.; Henderson, I.; Cooper, J.; Nayar, R.; Braun, L.J.; Carpenter, J.F. Stability of a trivalent recombinant protein vaccine formulation against botulinum neurotoxin during storage in aqueous solution. Journal of Pharmaceutical Sciences 2009, 98, 2970-2993.

4. Clapp, T.; Siebert, P.; Chen, D.; Jones Braun L.-T. Vaccines with Aluminum-containing Adjuvants: Optimizing Vaccine Efficacy and Thermal Stability. Journal of Pharmaceutical Sciences 2011, 100(2), 388-401.

5. Greiner, V.J.; Ronzon, F.; Larquet, E.; Desbat, B.; Estèves, C.; Bonvin, J.; Gréco, F.; Manin, C.; Klymchenko, A.S.; Mély, Y. The structure of HBsAg particles is not modified upon their adsorption on aluminium hydroxide gel. Vaccine 2012, 30(35), 5240-5245. doi: 10.1016/j.vaccine.2012.05.082.

6. Deshmukh, S.S.; Magcalas, F.W.; Kalbfleisch, K.N.; Carpick, B.W.; Kirkitadze, M.D. Characterization of TB vaccine components and study of structural changes of $\mathrm{H} 4$ antigen upon adjuvantation with IC $31^{\circledR}$. Journal of Pharmaceutical and Biomedical Analysis 2018, 157, 235-243. doi.org/10.1016/j.jpba.2018.05.048

7. Kirkitadze, M.; Arunachalam, A.; Carpick, B. Comparability of biotherapeutics: characterization of protein vaccine antigens. Pharmaceutical Bioprocessing 2013, 1(4), 373-380. doi:10.4155/PBP.13.42

8. De Mattia. F.; Chapsal, J.-M.; Descamps, J.; Halder, M.; Jarrett, N.; Kross, I.; Mortiaux, F.; Ponsar, C.; Redhead, K.; McKelvie, J.; Hendriksen C. The consistency approach for quality control of vaccines - A strategy to improve quality control and implement 3Rs. Biologicals 2011, 39 (1), 59-65.

9. Jones, L.S.; Peek, L.J.; Power, J.; Markham, A.; Yazzie, B.; Middaugh, C.R. Effects of adsorption to aluminum salt adjuvants on the structure and stability of model protein antigens. J. Biol Chem 2005, 280, 13406-13414.

10. Kirkitadze, M.; Sinha, A.; Hu, J.; Williams, W.; Cates, G. Adjuvanted vaccine components: Analysis of structure and stability. Procedia in Vaccinology 2009, 1, 135-139.

11. Zheng, Y,; Lai, X.; Ipsen, H.; Larsen, J.N.; Lowenstein, H.; Songergaard, I.; Jacobsen, S. The structural stability of protein antigens adsorbed by aluminum hydroxide in comparison to the antigens in solution. Spectroscopy 2007, 21, 257-268.

12. Peek, L.J.; Martin, T.T.; Elk Nation, C.; Pegram, S.A.; Middaugh, C.R. Effects of stabilizers on the destabilization of proteins upon adsorption to aluminum salt adjuvants. J Pharm Sci 2007, 96, 547-557. 
13. Olin, P. Commentary: The best acellular pertussis vaccines are multicomponent. Pediatric Infectious Diseases Journal 1997, 16(5), 517-519.

14. Andre, F.E. Development and clinical application of new polyvalent combined paediatric vaccines, Vaccine 1999, 17 (13-14), 1620-1627.

15. Capiau C, Poolman J, Hoet B, Bogaerts H, Andre F. Development and clinical testing of multivalent vaccines based on a diphtheria-tetanus-acellular pertussis vaccine: difficulties encountered and lessons learned. Vaccine 2003, 21: 2273-2287.

16. Gupta, R.K.; Rost, B.E. Aluminum compounds as vaccine adjuvants in Vaccine Adjuvants Preparation Methods and Research Protocols. Methods in Molecular Medicine, Ed. O'Hagan, D.T.; OHumana Press, Inc., Totowa, NJ., 2000, 42, pp. 65-89.DOI: 10.1385/1-59259-083-7:65

17. Sammons, R.; Marquis, P. Application of the low vacuum scanning electron microscope to the study of biomaterials and mammalian cells. Biomaterials 1997, 18, 81-86.

18. Quadracel Product Monograph https://www.vaccineshoppecanada.com/document.cfm?file=Quadracel_E.pdfQuadracel Product Monograph https://www.vaccineshoppecanada.com/document.cfm?file=Quadracel_E.pdf (accessed on 02 August 2018).

19. Pentacel Product Monograph http://novaccine.com/wp-content/uploads/2014/02/pentacel_E_package_insert.pdf (accessed on 02 August 2018)

20. 315 - Pediacel $®$ Product Monograph https://www.vaccineshoppecanada.com/document.cfm?file=pediacel_e.pdf (accessed on 02 August 2018).

21. Kirkitadze, M.D.; Carpick, B.W.; Williams, W.A.; Bhandal, K.S.; Kalbfleisch, K.N.; Deshmukh, S.S. Identification of Vaccine Products and Drug Substances by Fourier Transform Infrared (FTIR) Spectroscopy. Pharma Focus Asia, Knowledge Bank, 2018, July. https://www.pharmafocusasia.com/articles/identification-of-vaccine-products-and-drug-substances 